\title{
Monitoreo en línea de transformadores de potencia. Una revisión crítica de sensores de temperatura, humedad del aceite y gases disueltos
}

On-line monitoring of power transformers. A critical review of temperature sensors, oil moisture and dissolved gases

Santiago Salamanca ${ }^{1}$, Alexander Céspedes ${ }^{2}$, Guillermo Aponte ${ }^{3}$

Salamanca, S; Céspedes, A; Aponte, G. Monitoreo en línea de transformadores de potencia. Una revisión crítica de sensores de temperatura, humedad del aceite y gases disueltos. Tecnología en Marcha. Vol. 34, especial. ALTAE. Diciembre 2021. Pág 114-125.

doi) https://doi.org/10.18845/tm.v34i7.6019 


\title{
Palabras clave
}

Revisión crítica; monitoreo en línea; sensor; transformador de potencia; análisis de gases disueltos; humedad del aceite; temperatura.

\section{Resumen}

Los transformadores de potencia son uno de los activos más importantes que conforman las redes eléctricas. Cuando uno de estos activos presenta una falla repentina que involucra la suspensión del suministro de electricidad a los centros de consumo, puede conllevar consecuencias críticas para el sistema de potencia. La mayoría de este tipo de fallas en el transformador, están relacionadas con altos niveles de temperatura, humedad o deterioro de su sistema de aislamiento.

Debido a lo anterior, durante las últimas décadas los sistemas de monitoreo en línea para transformadores de potencia han venido adquiriendo una mayor relevancia e integración a las redes y subestaciones eléctricas. En este trabajo se realiza una revisión crítica de tres de los sensores en línea más importantes para la operación y funcionamiento del transformador, como lo son: temperatura, humedad del aceite y gases disueltos.

Inicialmente, se realiza una introducción referente a la importancia del transformador de potencia y el concepto de monitoreo en línea. Luego, se presenta una breve descripción con los principales hitos históricos de dichos sensores al igual que algunos de sus aspectos técnicos generales. Además, se proponen algunos criterios de comparación y selección citando ejemplos y casos estadísticos de interés. Finalmente, se presentan las conclusiones y recomendaciones del estudio.

\section{Keywords}

Critical review; online monitoring; sensor; power transformer; dissolved gas analysis; oil moisture; temperature.

\begin{abstract}
Power transformers are one of the most important assets that make up electrical networks. When one of these assets has a sudden failure that involves the suspension of electricity supply to consumption centers, it can have critical consequences for the power system. Most of these types of transformer failures are related to high levels of temperature, moisture or deterioration of its insulation system.

Due to the above, during the last decades online monitoring systems for power transformers have been acquiring greater relevance and integration to electrical networks and substations. In this work, a critical review of three of the most important online sensors for the operation and functioning of the transformer is carried out, such as: temperature, oil moisture and dissolved gases.

Initially, an introduction is made regarding the importance of the power transformer and the concept of online monitoring. Then, a brief description is presented with the main historical milestones of these sensors as well as some of their general technical aspects. In addition, some comparison and selection criteria are proposed, citing examples and statistical cases of interest. Finally, the conclusions and recommendations of the study are presented.
\end{abstract}




\section{Introducción}

Los transformadores son equipos importantes del sistema eléctrico de potencia, y debido a que su operación en la mayoría de los casos es permanente, representan un activo esencial para la red eléctrica. Por tanto, dicho elemento necesita ser monitoreado para garantizar su adecuado funcionamiento y prevenir posibles fallas, ya que esto implicaría consecuencias críticas como la pérdida de suministro de energía y repercusiones adicionales sobre los procesos productivos de las empresas o centros de servicios críticos como acueductos u hospitales. En adición, el transformador puede resultar dañado de manera parcial o total, produciendo un costo relevante por mantenimiento o adquisición de un nuevo equipo.

Debido a lo anterior, existen técnicas para determinar y predecir la condición de los transformadores de potencia, sin embargo, la mayoría de estas técnicas requieren desenergizar el equipo. Adicionalmente, el proceso de obtención e interpretación de los resultados no es inmediato, sino que toma un tiempo significativo desde su realización. Por otro lado, la alternativa del monitoreo en línea aparece como una opción conveniente y prometedora para mejorar la gestión de estos activos.

El objetivo principal de este trabajo consistió en realizar una revisión crítica de sensores en línea utilizados para el monitoreo de gases disueltos, humedad del aceite y temperatura de los transformadores de potencia. El documento inicia con la descripción de algunos conceptos fundamentales relacionados con los transformadores de potencia, las fallas a las que pueden estar sometidos y algunas de las técnicas de diagnóstico aplicadas. Luego, se revisan conceptos básicos del monitoreo en línea y los sensores bajo estudio, incluyendo sus principales características e hitos de desarrollo histórico. Más adelante, se realiza una discusión analítica basada en criterios propuestos para la comparación y selección de sensores en línea, enfatizando en algunos aspectos y casos estadísticos relevantes. Finalmente, se entregan las conclusiones del caso.

\section{Fallas en los transformadores de potencia}

El transformador de potencia es una máquina estática que introduce un acoplamiento mutuo entre circuitos eléctricos, permitiendo la transformación de niveles de tensión y de corriente por medio del principio de inducción electromagnética, manteniendo constantes los valores de frecuencia y de potencia. Además, es un elemento ampliamente utilizado en las diversas etapas del Sistema Eléctrico de Potencia (SEP), desde la generación hasta la distribución, y representa uno de los activos más importantes de la red eléctrica.

Los esfuerzos mecánicos, eléctricos y magnéticos, junto con los procesos de funcionamiento del transformador, producen ciertos efectos inherentes que tienen una repercusión negativa en el estado del equipo, bien sea a corto, mediano o largo plazo. Según [1], por medio de reportes y trabajos previamente realizados, se crearon estadísticas tomando como muestra cierta cantidad de transformadores de potencia y se registraron las fallas más comunes presentadas en sus componentes durante su tiempo de funcionamiento. La figura 1 muestra los principales elementos del transformador de potencia y su respectivo porcentaje de fallas. 

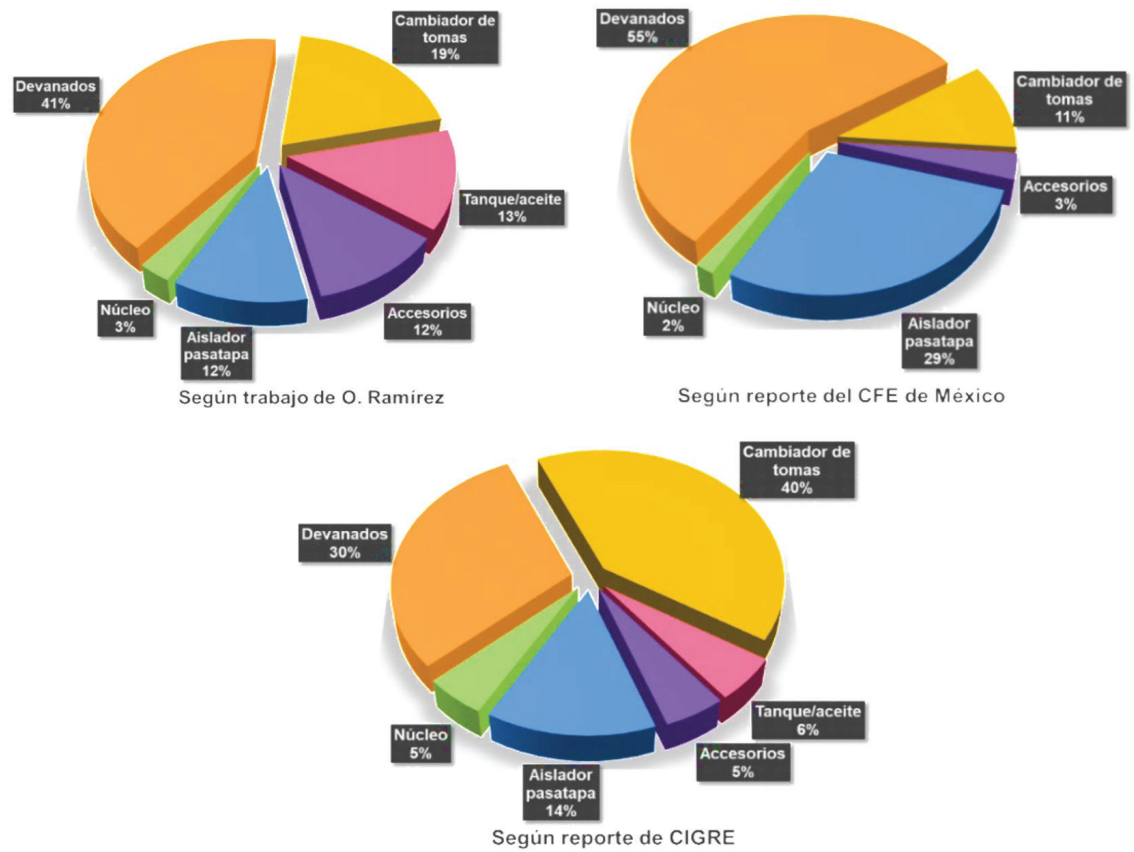

Figura 1. Principales elementos del transformador de potencia y su respectivo porcentaje de fallas. Fuente: adaptado de [1].

Como se observa en la figura 1, los reportes coinciden en que el sistema de devanados produce el mayor índice de fallas, seguido de componentes como el cambiador de tomas, los bujes (aislador pasatapa), el núcleo y el tanque.

Estos procesos de comparación permiten la creación de un sistema o base de datos que paulatinamente facilita posteriores análisis; de esta manera, la gestión y mantenimiento de los activos se convierte en una metodología más práctica y con un menor índice de error, detectando los elementos del transformador de potencia con mayores probabilidades de falla y permitiendo un monitoreo prioritario, pero sin descuidar los demás factores ni componentes operativos.

\section{Efectos debidos al calentamiento y la humedad}

La aparición de calor es una respuesta inherente en la funcionalidad del equipo; la temperatura aumenta progresivamente hasta alcanzar las condiciones de régimen permanente, punto en el cual permanece constante [2]. La elevación de la temperatura a valores que excedan los niveles de fabricación previamente establecidos, acelera la degradación de los materiales aislantes y, en consecuencia, acortan el período de vida útil del transformador.

Por ejemplo, para temperaturas entre los $300^{\circ} \mathrm{C}$ y los $700^{\circ} \mathrm{C}$, el sistema de aislamiento conformado por el aceite y papel aislante comienza a carbonizarse, disminuyendo la capacidad de rigidez dieléctrica [3]; durante este escenario también se generan gases disueltos en el aceite. Otro parámetro importante y que impacta directamente en la confiabilidad del activo es la presencia de humedad en el aislamiento sólido y líquido. La humedad dentro del transformador busca el equilibrio térmico, circulando entre el sistema aislante, la celulosa y el aceite; esta circulación es dependiente de la temperatura a la que está sometido el transformador. Por ejemplo, si se tienen altas temperaturas, la humedad tiende a migrar del papel al aceite, y a bajas temperaturas ocurre lo contrario [4]. Al juntarse los parámetros de humedad y temperatura, se produce el fenómeno de generación de burbujas, que se representa a través de vapor de agua y liberación de gases dentro del transformador [5]. 
Uno de los efectos directos de la generación de burbujas está relacionado con la reducción de la rigidez dieléctrica del líquido aislante; en adición, la humedad acelera el proceso de envejecimiento de la celulosa y produce esfuerzos mecánicos que expanden los aislamientos; estos efectos en conjunto, incrementan las probabilidades de una falla [5]. Por otro lado, las sobrecargas que se prolongan en el tiempo aumentan aún más la temperatura, resultando en un mayor calentamiento del transformador. Adicionalmente, el tamaño del transformador es un factor proporcional al efecto que tienen las sobrecargas; entre mayor sea el tamaño del equipo, mayor es el impacto que tiene este fenómeno sobre el activo, debido a que las fuerzas de cortocircuito y las densidades de flujo de dispersión son mayores, y el proceso de localización de puntos calientes es más complejo [3].

\section{Monitoreo en línea y diagnóstico de transformadores de potencia}

Teniendo en cuenta las potenciales condiciones de falla anteriormente descritas, las técnicas para conocer el estado y la condición del transformador de potencia han evolucionado gradualmente durante el transcurso de los años. A pesar de que su aplicación implica un adecuado seguimiento de la operación del activo, la mayoría de ellas presentan como requerimiento principal, la desconexión del equipo de la red eléctrica. Esta acción trae como consecuencia la parada obligatoria de los procesos de producción o en su defecto, la necesidad de otro equipo disponible para sustituir temporalmente al transformador bajo estudio.

A través de la evolución tecnológica de las últimas décadas, se han encontrado formas de utilizar elementos que faciliten la medición de los parámetros sin la necesidad de desconectar el transformador. El monitoreo en línea es un proceso que permite conocer aspectos de la condición del transformador de forma continua; su principal ventaja radica en la medición periódica de ciertos parámetros del equipo, sin la necesidad de generar una desconexión de la red eléctrica.

La implementación de este tipo de monitoreo debe basarse en la vigilancia de los parámetros clave relacionados al activo, con el fin de presentar los datos concernientes a la variable bajo estudio para facilitar su interpretación y análisis, y así obtener una alerta temprana de posibles incidentes. Lograr esta premisa a nivel práctico representa una disminución en la probabilidad de fallas. Por ejemplo, el diagnóstico y prevención a tiempo de posibles fallas, junto con el seguimiento de la tendencia de las variables hacen parte de estrategias de mantenimiento basado en la condición (MBC) de los transformadores. La figura 2 muestra algunos de los objetivos de este tipo de mantenimiento.

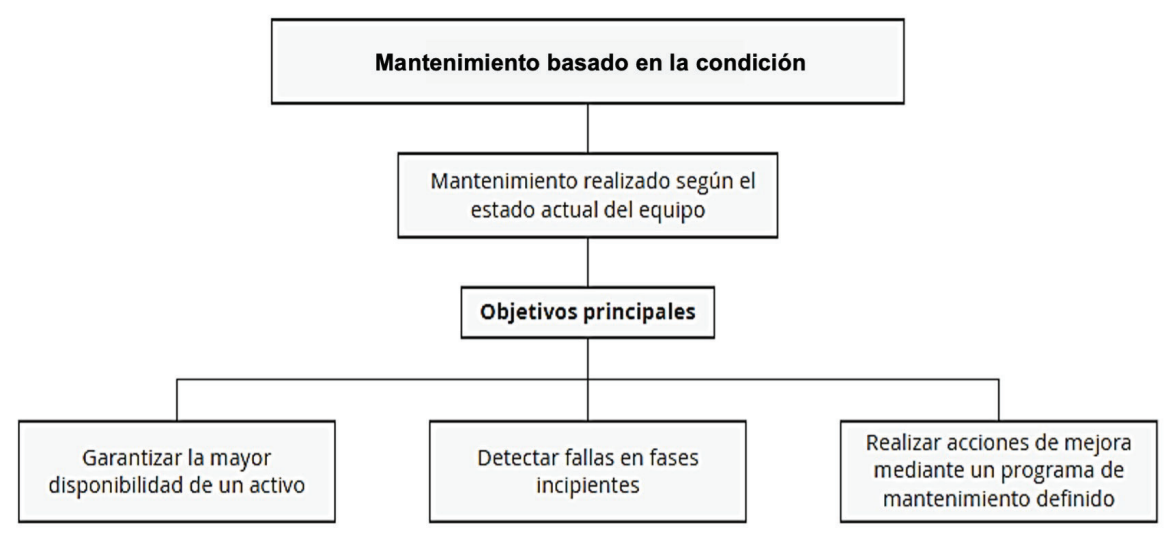

Figura 2. Objetivos fundamentales del mantenimiento basado en la condición para transformadores. 


\section{Tecnología en Marcha \\ Vol. 34, especial. Diciembre 2021 \\ Congreso de Alta Tensión y Aislamiento Electrónico

En términos generales, las ventajas del monitoreo en línea son resaltables, aunque existen limitantes como el hecho de que no todos los parámetros del transformador pueden ser medidos "en línea". Debido a esto, uno de los pilares importantes para el rendimiento del monitoreo en línea es el modelado adecuado de parámetros asociados a los datos históricos del equipo y el uso de herramientas de tipo estadístico. El modelado de parámetros es un trabajo preciso y que va estrechamente relacionado a un ámbito económico y técnico [6].

\section{Sensores en línea para transformadores de potencia}

En esta sección se mencionaran algunos aspectos históricos claves y características básicas relevantes relacionadas con los sensores en línea bajo estudio y las técnicas de medición que estos utilizan.

\section{Sensores de gases disueltos en aceite}

El análisis periódico de gases disueltos en el aceite (DGA por sus siglas en inglés), es ampliamente utilizado para obtener información significativa sobre el estado del sistema de aislamiento de un transformador a partir de la interpretación de las concentraciones de gases. Por tanto, la aplicación del monitoreo en línea de gases disueltos reduce considerablemente el riesgo de no detectar posibles fallas a tiempo debido a los intervalos considerables de tiempo de muestreo del aceite en otras alternativas, como por ejemplo, el envío y análisis a un laboratorio [7]. Una de las características relevantes en el DGA, es que el comportamiento de gasificación tiende a ser único para cada transformador, pues la concentración de los gases dependerá de factores como la antigüedad, las condiciones de operación y las características internas; por tanto, el enfoque de estudio debe centrarse en las tendencias y tasas de cambio de los gases [8].

Los primeros indicios de este método se sitúan en la década de 1960 y numerosos estudios y experimentos se han realizado con la finalidad de perfeccionarlo a lo largo del tiempo [9]. En 1970, Dornenburg propone una técnica para diferenciar la naturaleza de las fallas y así establecer si su origen era térmico o eléctrico [10]. Más adelante, se determinó que la presencia de gases combustibles depende de la temperatura en el aceite del transformador; al presentarse una falla, la temperatura del transformador aumenta y, en consecuencia, se generan cierta cantidad de gases en la cuba del activo. Mediante este concepto, en el año de 1974, se establece la técnica de Gases Claves [10].

Cuatro años más tarde, en 1978, Rogers determina que la concentración de ciertos gases (etileno y acetileno) requieren de una temperatura elevada para generarse; igualmente, introduce nuevas relaciones entre las proporciones de gases [10]. Un tiempo después, importantes instituciones normativas como la IEEE y la IEC presentaron diversos estándares relacionados al análisis de los gases disueltos; en 1991 se publica el estándar C57.104.1991 "Guía para la interpretación de gases generados en transformadores inmersos en aceite", así mismo, la IEC publica la guía IEC 60599 para la interpretación de los gases disueltos. Estas guías han tenido varias actualizaciones en los últimos veinte años [11].

En el año 2002, Duval interpreta los resultados de varios estudios basados en gases disueltos mediante métodos gráficos, estableciendo seis tipos de fallas principales según el tipo de gas generado. Esta técnica es conocida como el Triángulo de Duval [9]. En 2006, J.L. Ganesha reporta los datos experimentales de los efectos que tienen el arco eléctrico sobre la generación de gases disueltos y el líquido aislante del transformador [10].

Ya en el año 2010, se demuestra la compatibilidad y aplicabilidad del análisis de gases disueltos mediante sistemas de monitoreo en línea; además, se determina que la unión de esta técnica convencional y los beneficios que representa el monitoreo en línea permiten la 
detección de hasta un 70\% de las fallas más comunes en los transformadores de potencia [10]. Durante la última década, se han creado algunas metodologías para la validación de datos y funcionamiento de los monitores de DGA en línea [12]. En 2019, se desarrolló un estudio para determinar un sensor que realizara mediciones sin contacto; así, se estudió la detección de múltiples gases con sensores basados en el espectro de absorción láser de diodo sintonizable TDLAS [13], utilizando técnicas fisicoquímicas y ópticas.

\section{Sensores de humedad en el aceite}

Como se mencionó anteriormente, los niveles excesivos de humedad inciden en forma negativa sobre la operación del transformador, afectando principalmente la rigidez dieléctrica y la vida útil del aislamiento junto con la capacidad de carga dicho equipo.

A nivel histórico, se puede mencionar que en el año de 1935, el químico alemán Karl Fischer creó un método para determinar el contenido de agua y humedad en una muestra específica de aceite, conocido como el método de titulación de Karl Fischer (KFT), el cual es realizado en laboratorio. Con el paso del tiempo se ha determinado que este proceso de medición presenta ciertas limitaciones en su precisión y una probabilidad importante de contaminación de la muestra de aceite. Estas mismas limitaciones motivaron la búsqueda de nuevas tecnologías para el monitoreo de la humedad.

Varias décadas después, Oommen probó sensores de humedad capacitivos de película fina en el año de 1991 [14]. En dicho estudio, se determinó que este tipo de sensores respondían bien ante las variaciones de humedad y temperatura [15]. Adicionalmente, durante estos años, se proponen algunas tecnologías alternativas tales como sensores con sales higroscópicas, sensores psicrométricos y sensores basados en polímeros. En los últimos tiempos, los sensores de fibra óptica para medición en línea de humedad en el aceite han sido evaluados [16].

Algunos estudios relevantes en este sentido son los sensores de fibra óptica basados en un sistema de microcontroladores y algoritmos de redes neuronales artificiales ANN [17] y los sensores de fibra óptica basados en redes de Bragg FBG [18], entre otras tecnologías propuestas. En la Figura 3 se muestra un ejemplo de una línea de tiempo con los principales hitos en relación a la técnica de medición de humedad en el aceite.

\section{Sensores de temperatura}

El aumento de temperatura o el sobrecalentamiento puede causar fallas en el transformador. En la norma IEC-60076-7 [19], sección 7, se establece que "la tasa de envejecimiento térmico es proporcional a la temperatura del punto caliente del devanado cada $6{ }^{\circ} \mathrm{C}$ aumenta el envejecimiento a base de hidrólisis por el doble".

Adicionalmente, los materiales aislantes pierden su resistencia mecánica debido a la exposición prolongada a altas temperaturas, provocando además posibles desgarros y desplazamientos del papel aislante, lo que aumenta las probabilidades de una falla dieléctrica prematura [20].

El impacto de la temperatura sobre la condición de los transformadores de potencia ha sido un fenómeno estudiado desde principios del siglo pasado. Varios organismos internacionales han venido desarrollando estándares donde se mencionan los efectos de las altas temperaturas y el punto caliente en los devanados del activo IEEE C57.91 de 1981 e IEC 60354 de 1991. En ese sentido, el método de medición directa de temperatura ha venido mostrando resultados positivos en comparación a la medición indirecta. 


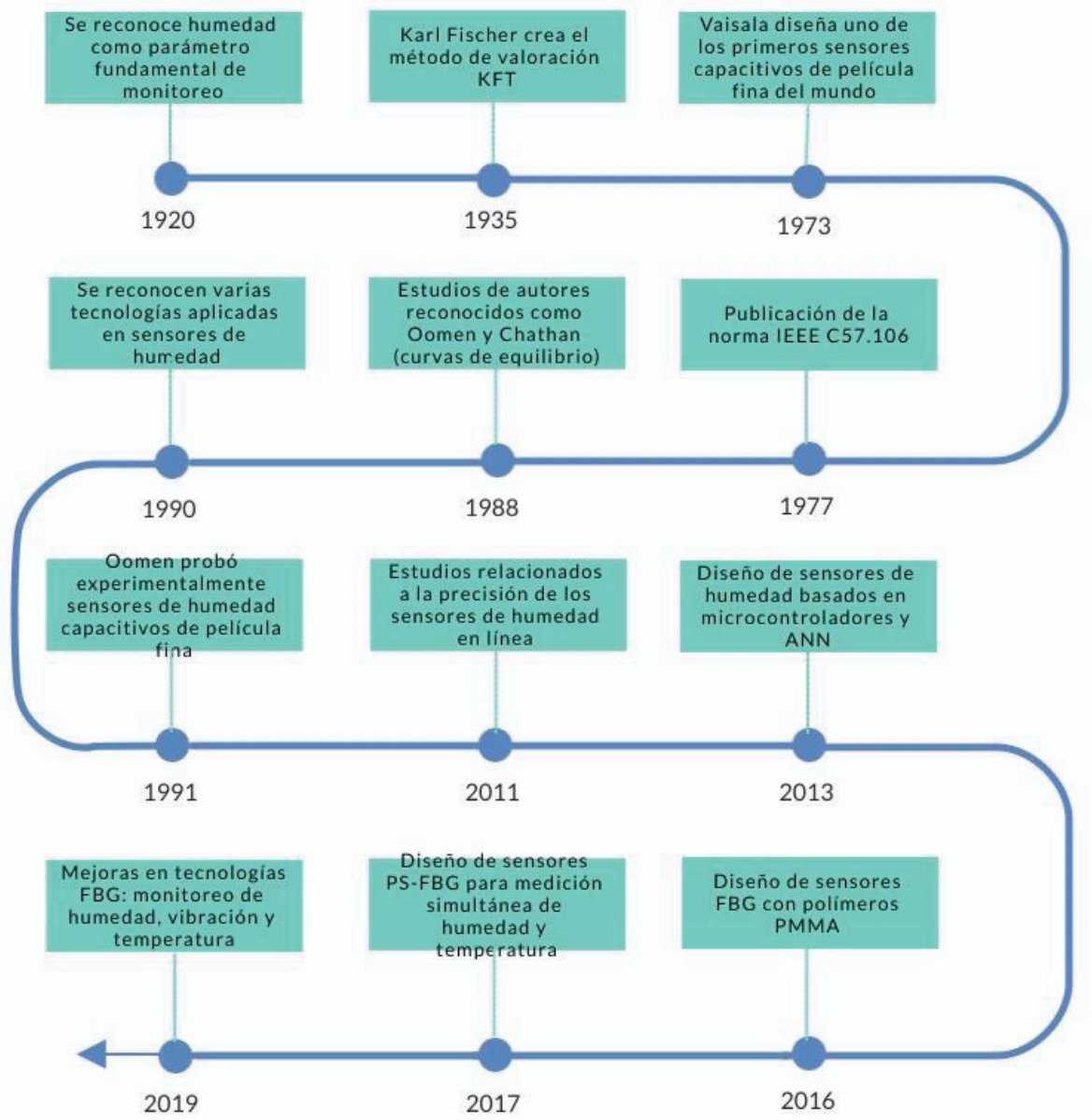

Figura 3. Línea de tiempo con los principales hitos de la medición de humedad en el aceite de transformador.

Dicho método consiste en la integración de sensores en el transformador para medir las temperaturas internas del equipo obteniendo resultados más confiables, al determinar el valor de temperatura en función del estado actual del transformador [21], y no basado únicamente en ecuaciones y modelos térmicos predeterminados [22]. Actualmente, la medición directa puede realizarse mediante señales eléctricas, infrarrojas y ópticas [23].

En concordancia a lo descrito anteriormente, los sensores de fibra óptica para la medición de temperatura han venido ganando popularidad durante los últimos 20 años [24], y han generado gran interés por su rápida respuesta de sensado y baja susceptibilidad a las perturbaciones externas. Sin embargo, desde el año 2018 se ha venido observando la degradación y envejecimiento acelerado en las fundas de la fibra óptica que se instalan dentro del transformador.

Este efecto se atribuye a la exposición continua a altas temperaturas y a las reacciones químicas, afectando la medición del sensor y contaminando el aislamiento líquido a causa del material residual debido al desgaste de las fundas. Es por ello, que últimamente se han venido liderando estudios para mejorar los recubrimientos de la fibra óptica, evaluando materiales más resistentes y sus tasas de envejecimiento térmico [25]. 


\section{Discusión final}

Luego de describir algunos de los hitos históricos y características más relevantes alrededor de las técnicas de diagnóstico y sensores en línea de DGA, humedad del aceite y temperatura, los autores consideran importante realizar una breve discusión teniendo en cuenta algunos criterios que pueden utilizarse para la comparación y selección de dichos sensores, tales como:

1. Tecnología utilizada: relaciona el tipo de tecnología de medición usada por el sensor.

2. Especificaciones de medida: basadas en el rango, la precisión y exactitud del sensor.

3. Especificaciones de comunicación: involucra los protocolos de comunicación e interfaces de usuario utilizadas por el sensor.

4. Especificaciones físicas: tiene que ver con las dimensiones, peso e índice de protección (IP) del sensor.

5. Especificaciones ambientales y de operación: tiene en cuenta características de temperatura ambiente, humedad relativa y régimen de trabajo soportables por el sensor.

Según los criterios anteriores, se pueden estudiar ciertos parámetros importantes en relación a cada sensor con el fin de garantizar la adecuada selección de los mismos según el caso del transformador a instrumentar. Por ejemplo, para los sensores de DGA las especificaciones relacionadas a la medición de gases de los monitores brinda un marco comparativo que puede facilitar la elección de uno sobre otro, en caso de que se requiera una precisión alta, un rango de detección elevado, o si se permite un margen de error para ciertas aplicaciones.

Entre los sensores de DGA revisados en este estudio (treinta en total), se encontró que todos los monitores de gas simple miden el hidrógeno, la mayoría incluyen la opción de medición de humedad en el aceite del transformador, y una menor cantidad incluye la medición del monóxido de carbono. Por otra parte, algunos de los sensores tienen disponible la medición de otros gases como el acetileno $\mathrm{C} 2 \mathrm{H} 2$ y el etileno $\mathrm{C} 2 \mathrm{H} 4$.

Adicionalmente, se realizó el mismo estudio estadístico en los monitores multigas. La Figura 4 presenta los resultados de dicho estudio.

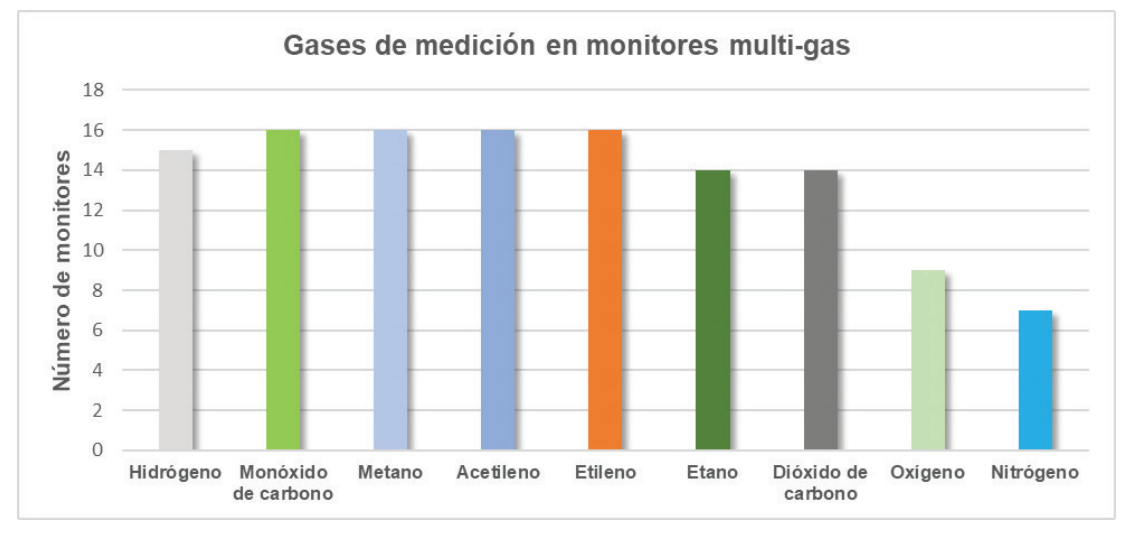

Figura 4. Gases típicos medidos en monitores DGA tipo multigas bajo estudio.

Otro ejemplo interesante encontrado, fue a nivel de los sensores de humedad del aceite, en donde al revisar las principales fichas técnicas, se observó que existe un protocolo de comunicación mayoritariamente aplicado entre la población bajo estudio. La Figura 5 contiene el comparativo estadístico. 


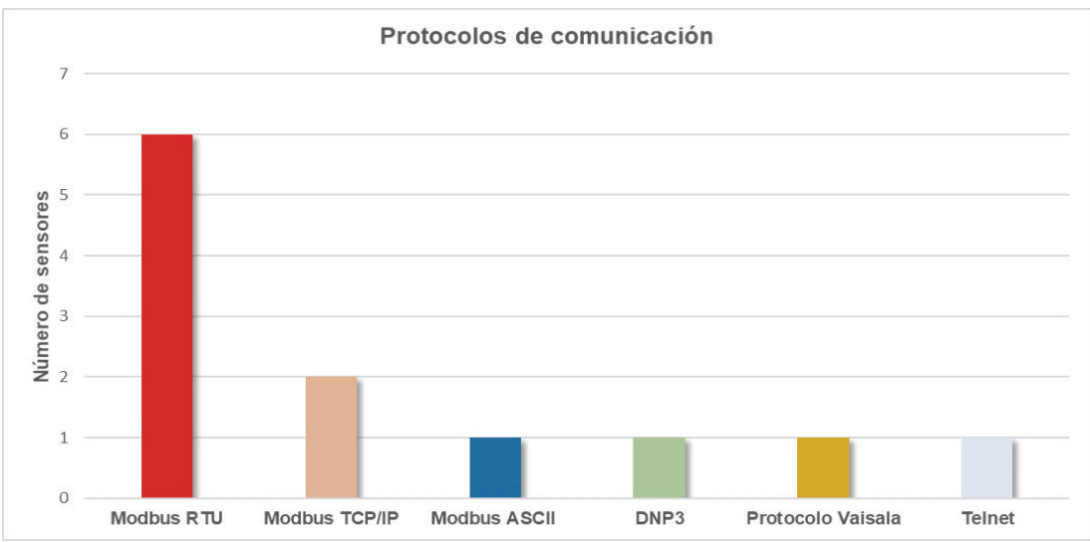

Figura 5. Protocolos de comunicación utilizados en monitores DGA tipo multigas bajo estudio.

Como se puede ver en la figura anterior, el protocolo Modbus RTU es el más aplicado entre la población de equipos para medición en línea de humedad en el aceite, y esto puede atribuirse a las características de dicho protocolo, el cual basa su funcionamiento en el intercambio de datos entre dos puntos. Su arquitectura de red está conformada por un equipo maestro y uno o más equipos esclavos; permitiendo una fácil integración de los dispositivos que lo usan en procesos industriales.

Por otro lado, una de las características relevantes a nivel de sensores de temperatura y que están relacionadas con el tipo de tecnología, en este caso fibra óptica, es el número de canales de medición utilizados. El número de canales de medición representa el número de sensores de fibra óptica que pueden utilizarse para monitorear la temperatura del transformador en cualquier punto. En este caso se incluyeron dentro del estudio siete equipos a nivel comercial, arrojando los resultados mostrados en la figura 6.

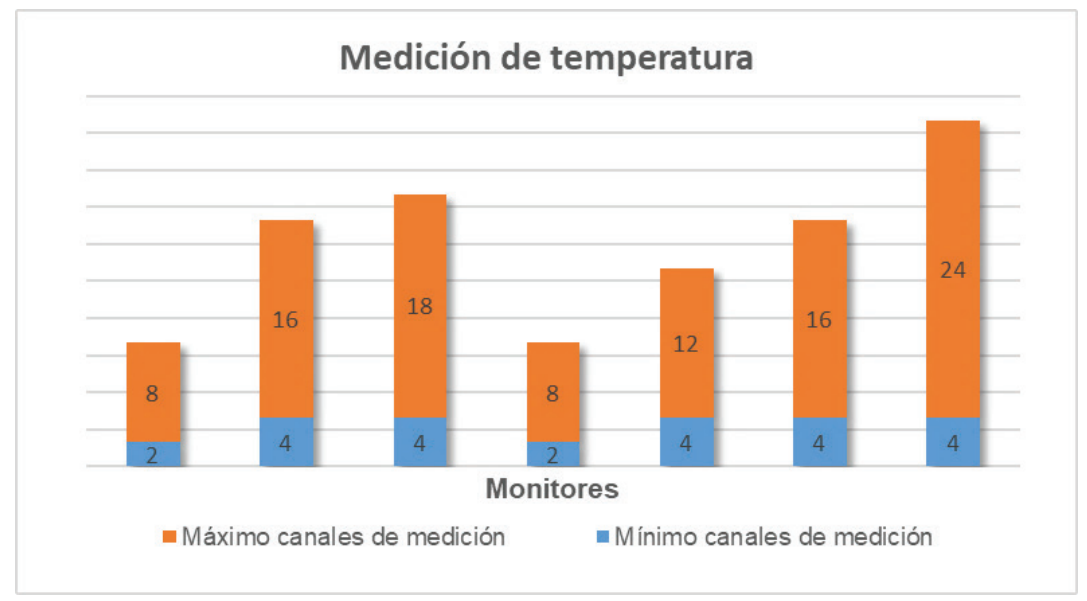

Figura 6. Número de canales de medición en los monitores de temperatura bajo estudio.

Como se observa en la figura 6, existen monitores con un mayor número máximo de canales que otros. Esta característica podría ser un punto decisivo de elección. Además, para ciertos casos de monitoreo, el número de canales de medición puede no ser un factor opcional, sino un requerimiento. Por ejemplo, los transformadores de potencia más grandes o con un mayor número de devanados, requieren, por obvias razones, de una mayor cantidad de sensores para cubrir una mayor área con el fin de estimar adecuadamente el modelo térmico del activo. 
Finalmente, se podría pensar que la solución más adecuada es aquel sensor que tenga el mejor rendimiento y se destaque en la mayoría de criterios en el grupo de estudio. Por ejemplo, la adquisición de un sensor multigas avanzado, un sensor de humedad con precisión e índice de protección considerable o un monitor de temperatura con el máximo número de canales, rango de medida e interfaz de usuario amigable.

Sin embargo, es importante resaltar que a un nivel práctico, generalmente se tiene definido un presupuesto máximo para los procesos de mantenimiento del activo y, por tal motivo, no siempre es viable la alternativa que se considere más completa como la primera opción. Por tanto, es importante realizar al menos un proceso de selección preliminar que justifique los costos de inversión y garantice que, bajo las necesidades de cada usuario, se realice el monitoreo adecuado del transformador de potencia.

\section{Conclusiones}

Dentro de las estrategias de mantenimiento aplicadas a los transformadores de potencia, es importante tener en cuenta factores como la ubicación geográfica del activo, las condiciones operativas, su nivel de criticidad en la red eléctrica y el nivel de automatización y monitoreo que se requiere implementar en el mismo. Algunas de las principales técnicas para conocer la condición del transformador de potencia, ya han sido integradas a las tecnologías de medición en línea tales como análisis de gases disueltos, humedad del aceite y temperatura del transformador.

A través del recorrido histórico realizado, se puede apreciar que la integración en el tiempo de técnicas convencionales potencializadas con los beneficios que representa el monitoreo en línea, brindan como resultado herramientas más confiables y versátiles para el estudio de la condición del transformador de potencia. Además, existen características específicas en los sensores en línea que relacionan aspectos como la medición de parámetros, su comunicación, características físicas, de protección y límites ambientales y operativos, ofreciendo una mayor personalización y funcionalidad para aquellos sistemas que los integren.

En otras palabras, lo que puede apreciarse es que existe una mayor posibilidad de encontrar sensores en el mercado que se adecuen a las necesidades y requerimientos de los usuarios o empresas del sector eléctrico, lo que incentiva la aplicación de alternativas de monitoreo en línea en transformadores de potencia.

\section{Agradecimientos}

Los autores desean agradecer a la Universidad del Valle y al Grupo de Investigación en Alta Tensión (GRALTA) por su apoyo para el desarrollo y divulgación del presente estudio.

\section{Referencias}

[1] R. Pérez, H. Torrez, E. Fernández, and S. Fernández, "Sistema de Monitoreo en Tiempo Real para el Diagnóstico de Transformadores de Potencia en una Empresa de Energía”. Lat. Am. Caribb. Conf., no. 2, p. $11,2012$.

[2] J. Carcedo, "Aportaciones al estudio del envejecimiento de componentes dieléctricos en transformadores de potencia". Tesis de Grado. Universidad Carlos III de Madrid. Madrid, España. 2015.

[3] R. SAIZ, "Análisis de la salud de una flota de transformadores industriales". Tesis de Grado. Universidad de Cantabria. Santander, España. 2017.

[4] CIGRE Working Group A2.37. "Transformer Reliability Survey”. Paris. Diciembre. 2015. 
[5] R. L. García, D. P. Noyola, A. G. López, and E. B. Ramírez. "Modelos para el monitoreo de transformadores de potencia en la red eléctrica inteligente : humedad y temperatura de generación de burbujas". Boletín IEE. pp. 3-10. México. 2013.

[6] R. Guo, X. Yan, Q. Peng, Y. Cao, and H. Zhang, "Research of pre-warning and diagnosis for transformer based on on-line monitoring devices," IEEE 2013 Tencon - Spring, TENCONSpring 2013 - Conf. Proc., pp. 381-385, 2013, doi: 10.1109/TENCONSpring.2013.6584476.

[7] D. Chu and A. Lux, "On-line monitoring of power transformers and components: A review of key parameters," Proc. - Electr. Insul. Conf. Electr. Manuf. Coil Wind. Conf. EEIC 1999, pp. 669-675, 1999, doi: 10.1109/ EEIC.1999.826290.

[8] P. Guy and B. Sparling, "Transformer asset life extension - When, why and how to apply continuous condition monitoring systems," IET Conf. Publ., vol. 2018, no. CP757, pp. 779-784, 2018, doi: 10.1049/cp.2018.1821.

[9] V. Kirylchyk Yevmenchik, "Aplicación de la metodología de cálculo de índice de salud de activos a transformadores de potencia. Propuestas de mejora". p.94.2017. https://idus.us.es/xmlui/handle/11441/69380.

[10] J. T. Sarria-Arias, N. A. Guerrero-Bello, and E. Rivas-Trujillo, "Estado del arte del análisis de gases disueltos en transformadores de potencia," Rev. Fac. Ing., vol. 23, no. 36, p. 105, 2014, doi: 10.19053/01211129.2716.

[11] J.D. Zorrilla, "Revisión crítica de las técnicas usadas para el diagnóstico de la condición de transformadores de potencia,". Tesis de Grado, pp. 1-14 Universidad del Valle. Cali, Colombia. 2017.

[12] Z. Zhou et al., "Validity Evaluation Method of DGA Monitoring Sensor in Power Transformer Based on Chaos Theory," Annu. Rep. - Conf. Electr. Insul. Dielectr. Phenomena, CEIDP, vol. 2018-Octob, pp. 402-405, 2018, doi: 10.1109/CEIDP.2018.8544854.

[13] J. Jiang et al., "Multi-gas detection in power transformer oil based on tunable diode laser absorption spectrum," IEEE Trans. Dielectr. Electr. Insul., vol. 26, no. 1, pp. 153-161, 2019, doi: 10.1109/TDEl.2018.007535.

[14] T. V. Oommen, "On-line moisture sensing in transformers". Proceedings of the 20th Electrical Electronics Insulation Conference, 1991, pp. 236-240, doi: 10.1109/EEIC.1991.162613.

[15] T. K. Saha, "Review of Modern Diagnostic Techniques for Assessing Insulation Condition in Aged Transformers". IEEE Trans. Dielectr. Electr. Insul., vol. 10, no. 5, pp. 903-917, 2003, doi: 10.1109/TDEl.2003.1237337.

[16] G. L. G. Pavan Kumar et al., "Modelling of Polymer Coated-based Optical Fiber Sensor for Moisture Measurement in Transformer Oil". ICECOS 2019 - 3rd Int. Conf. Electr. Eng. Comput. Sci. Proceeding, pp. 45-50, 2019, doi: 10.1109/ICECOS47637.2019.8984502.

[17] S. Laskar and S. Bordoloi, "Monitoring of Moisture in Transformer Oil Using Optical Fiber as Sensor". J. Photonics, vol. 2013, pp. 1-7, 2013, doi: 10.1155/2013/528478.

[18] W. Zhang and D. J. Webb, "PMMA based optical fiber bragg grating for measuring moisture in transformer oil". IEEE Photonics Technol. Lett., vol. 28, no. 21, pp. 2427-2430, 2016, doi: 10.1109/LPT.2016.2598145.

[19] IEC, "Loading guide for oil-immersed power transformers," IEC, Geneva, Standard IEC 60076-7, 2018.

[20] IEEE. "IEEE Guide for Application for Monitoring Equipment to Liquid-Immersed Transformers and Components". IEEE Std C57.143-2012, vol., no., pp.1-83, 19 Dec. 2012, doi: 10.1109/IEEESTD.2012.6387561.

[21] W. G. Chen, J. Liu, Y. Y. Wang, L. M. Liang, J. B. Zhao, and Y. F. Yue, "The measuring method for internal temperature of power transformer based on FBG sensors," 2008 Int. Conf. High Volt. Eng. Appl. ICHVE 2008, pp. 672-676, 2008, doi: 10.1109/ICHVE.2008.4774024.

[22] I. Hurezeanu, C. I. Nicola, D. Sacerdotianu, M. Nicola, A. M. Aciu, and M. C. Nitu, "Temperature control and monitoring system for power transformer windings using fiber optic sensors". 2016 Int. Symp. Fundam. Electr. Eng. ISFEE 2016, 2016, doi: 10.1109/ISFEE.2016.7803151.

[23] X. Hou, Dan, ZHANG, W. HUANG, M. ZHENG, and S. YAO, "Oil-immersed transformer online hot spot temperature monitoring and accurate life loses calculation based on fiber Bragg grating sensor techonology". Ciced, pp. 23-26, 2014.

[24] P. Lu et al., "Real-Time Monitoring of Temperature Rises of Energized Transformer Cores with Distributed Optical Fiber Sensors," IEEE Trans. Power Deliv., vol. 34, no. 4, pp. 1588-1598, 2019, doi: 10.1109/ TPWRD.2019.2912866.

[25] Y. Liu, S. Jiang, X. Fan, and Y. Tian, "Effects of degraded optical fiber sheaths on thermal aging characteristics of transformer oil," Appl. Sci., vol. 8, no. 8, 2018, doi: 10.3390/app8081401. 